\title{
MRI Findings in Children with Acute Flaccid Paralysis and Cranial Nerve Dysfunction Occurring during the 2014 Enterovirus D68 Outbreak
}

\author{
J.A. Maloney, D.M. Mirsky, K. Messacar, S.R. Dominguez, T. Schreiner, and N.V. Stence
}

\begin{abstract}
BACKGROUND AND PURPOSE: Enterovirus D68 was responsible for widespread outbreaks of respiratory illness throughout the United States in August and September 2014. During this time, several patients presented to our institution with acute flaccid paralysis and cranial nerve dysfunction. The purpose of this report is to describe the unique imaging findings of this neurologic syndrome occurring during an enterovirus D68 outbreak.
\end{abstract}

MATERIALS AND METHODS: Patients meeting a specific case definition of acute flaccid paralysis and/or cranial nerve dysfunction and presenting to our institution during the study period were included. All patients underwent routine MR imaging of the brain and/or spinal cord, including multiplanar T1, T2, and contrast-enhanced T1-weighted imaging.

RESULTS: Eleven patients met the inclusion criteria and underwent MR imaging of the brain and/or spinal cord. Nine patients presented with brain stem lesions, most commonly involving the pontine tegmentum, with bilateral facial nerve enhancement in 1 patient. Ten patients had longitudinally extensive spinal cord lesions; those imaged acutely demonstrated involvement of the entire central gray matter, and those imaged subacutely showed lesions restricted to the anterior horn cells. Ventral cauda equina nerve roots enhanced in 4 patients, and ventral cervical nerve roots enhanced in 3 , both only in the subacute setting.

CONCLUSIONS: Patients presenting with acute flaccid paralysis and/or cranial nerve dysfunction during the recent enterovirus D68 outbreak demonstrate unique imaging findings characterized by brain stem and gray matter spinal cord lesions, similar to the neuroimaging findings described in previous outbreaks of viral myelitis such as enterovirus 71 and poliomyelitis.

ABBREVIATIONS: AFP = acute flaccid paralysis; EV-D68 = enterovirus D68; EV-71 = enterovirus 71

$\mathrm{H}$ uman enteroviruses are ubiquitous pathogens throughout the world and cause a variety of disease states, including respiratory infections, herpangina, hand-foot-and-mouth disease, and aseptic meningitis. Enterovirus D68 (EV-D68), first identified in California in 1962, ${ }^{1}$ has been described primarily as a cause of respiratory illness. ${ }^{2,3}$ More recently, however, rare cases of CNS disease have been attributed to EV-D68. ${ }^{4,5}$

In August and September 2014, EV-D68 was responsible for widespread outbreaks of respiratory disease throughout the

Received October 15, 2014; accepted after revision October 25.

From the Departments of Radiology (I.A.M., D.M.M., N.V.S.) and Pediatrics, Section of Pediatric Infectious Disease (K.M., S.R.D.), Section of Hospital Medicine (K.M.), and Section of Child Neurology (T.S.), Children's Hospital Colorado, University of Colorado School of Medicine, Aurora, Colorado.

J.A. Maloney and D.M. Mirsky are co-first authors of this article.

Please address correspondence to John A. Maloney, MD, Department of Radiology, Children's Hospital Colorado, University of Colorado School of Medicine, 13123 E 16th Ave, Aurora, CO 80045; e-mail: john.maloney@childrenscolorado.org

- Indicates open access to non-subscribers at www.ajnr.org

三 Indicates article with supplemental on-line table.

http://dx.doi.org/10.3174/ajnr.A4188
United States. ${ }^{6}$ Against this backdrop, Children's Hospital Colorado noted an unusual number of cases of acute flaccid paralysis (AFP) and cranial nerve dysfunction following a febrile upper respiratory illness. This association led the Centers for Disease Control and Prevention to issue a national Health Advisory, ${ }^{7}$ and it was subsequently described in the Morbidity and Mortality Weekly Report. ${ }^{8}$

Enteroviruses have been associated with neurologic syndromes previously, most notably enterovirus 71 (EV-71). EV-71 most commonly presents with nonneurologic manifestations but has been associated with multiple outbreaks of AFP and brain stem encephalitis throughout the world. ${ }^{9-15}$ MR imaging in affected patients typically reveals a rhombencephalitis affecting the dorsal pons and medulla, and a radiculomyelitis with a predilection for the anterior horn cells of the spinal cord and ventral nerve roots, findings that have also been described in poliomyelitis. ${ }^{16-19}$ Although EV-D68 has not been proved as the causative agent in this cluster, these cases demonstrate distinctive imaging features that are very similar to the neuroimaging presentation of both EV-71 and poliovirus. 

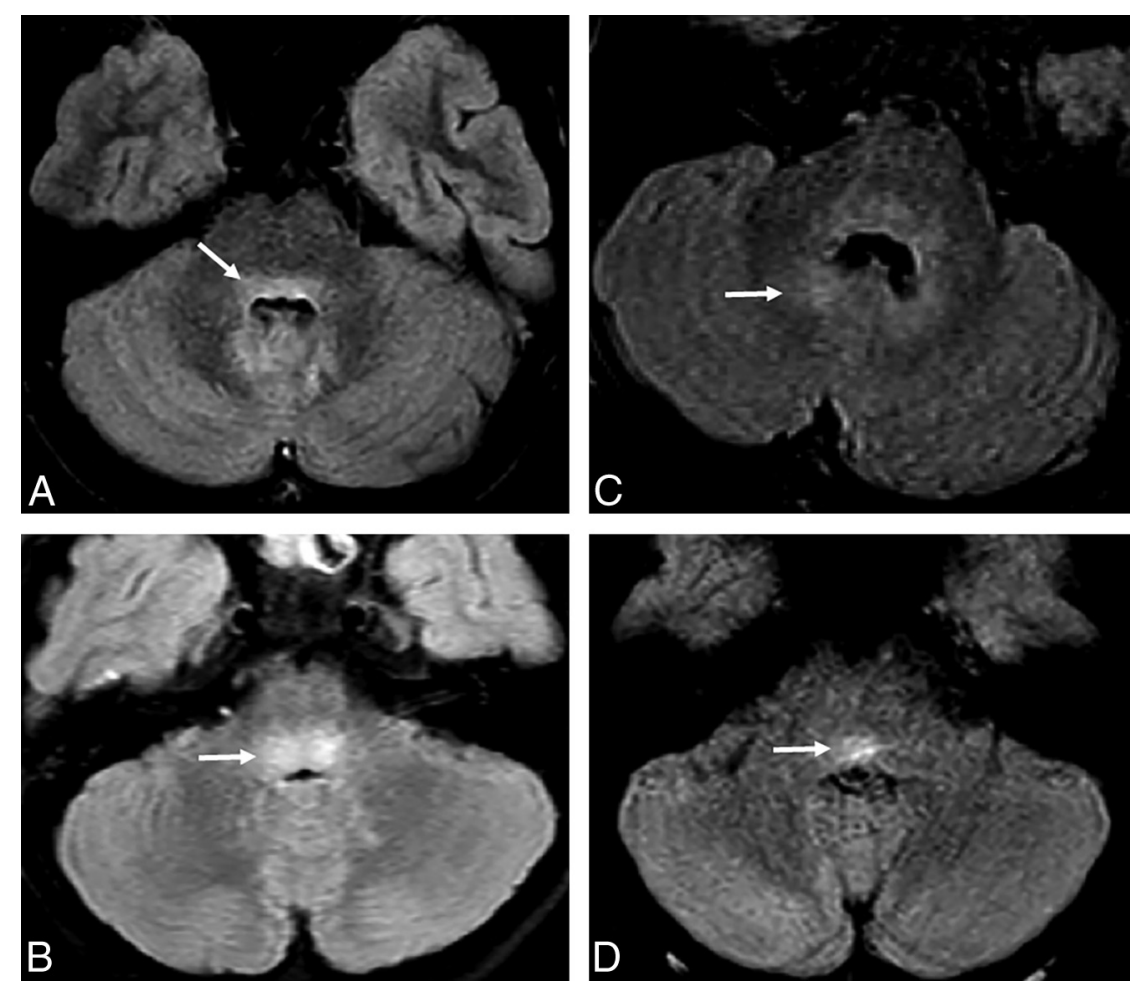

FIG 1. Noncontrast FLAIR images through the dorsal pons demonstrate a variety of types of T2 hyperintensity. $A$, In patient 4, T2 hyperintensity in the dorsal pons (arrow) extends into the dentate nuclei. $B$, In patient 2 , more focal and intense T2 signal is localized in the dorsal pons (arrow). C, In patient 6, ill-defined and less intense signal in the dorsal pons extends to the dentate nuclei (arrow). $D$, In patient 10, more focal and T2 hyperintensity is seen within the dorsal pons (arrow).
We present an imaging-based report describing in detail the MR imaging findings of neurologic diseases associated with an EV-D68 outbreak.

\section{MATERIALS AND METHODS}

\section{Patients}

Patients who presented to our institution from August 1, 2014, to October 1, 2014, with symptoms of AFP and/or cranial nerve dysfunction and with lesions in the brain stem or spinal cord gray matter on MR imaging were included.

\section{MR Imaging}

Imaging was performed on a $1.5 \mathrm{~T}$ or $3 \mathrm{~T}$ Ingenia (Philips Healthcare, Best, the Netherlands) or a 1.5T Avanto unit (Siemens, Erlangen, Germany). Ten of 11 patients underwent MR imaging of the brain, 7 had MR imaging of the entire spine, 2 had MR imaging of the cervical and thoracic spine only, and 1 had MR imaging of the cervical spine only. One patient did not undergo dedicated spine imaging. Parameters and specific sequences of various MR imaging brain examinations were variable, though each patient underwent isometric 3D T1-weighted gradient-echo imaging be-
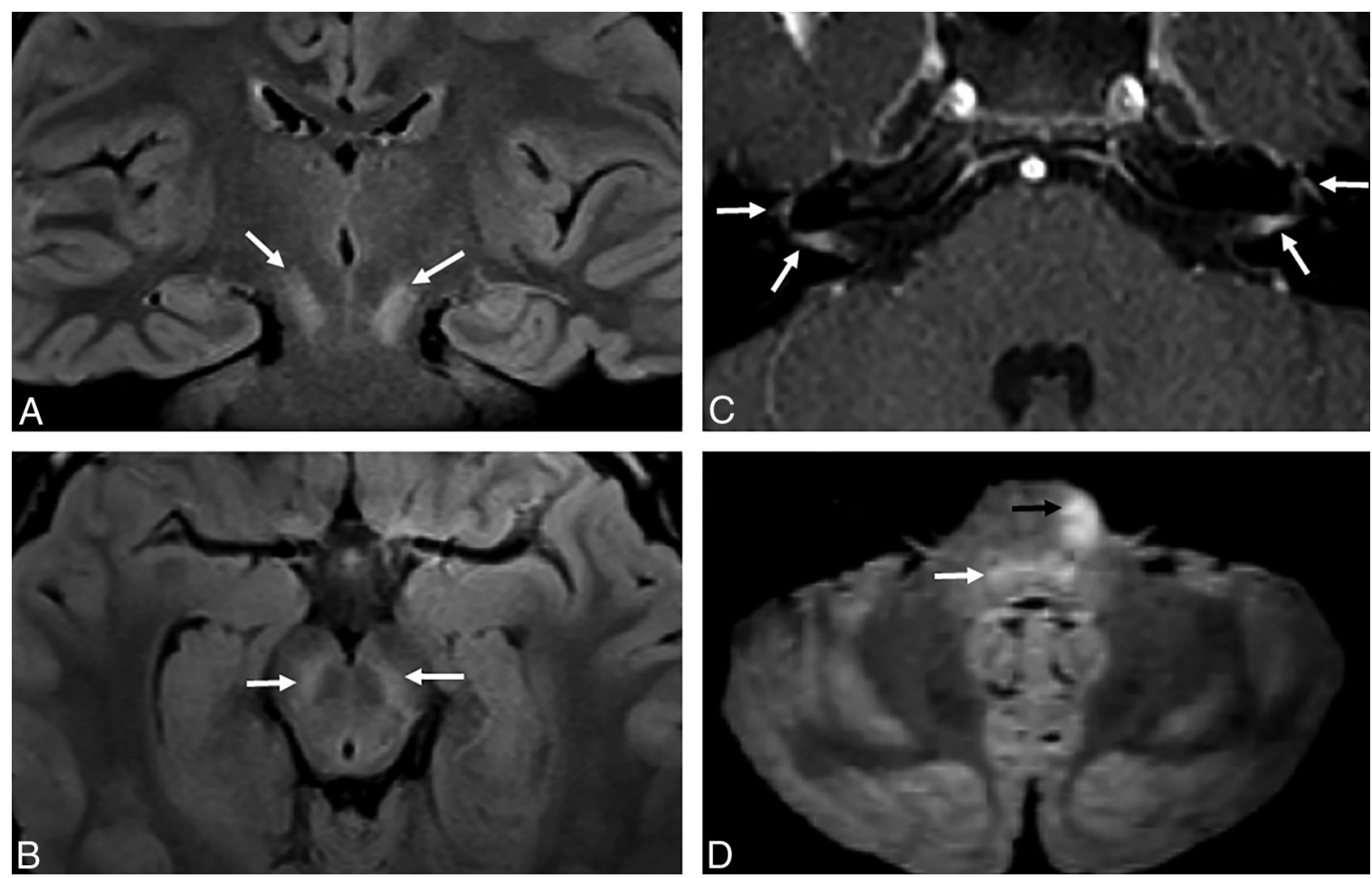

FIG 2. Atypical appearance of CNS disease includes T2 hyperintensity localized to the substantia nigra in patient 3 (white arrows, $A$ and $B$ ). In patient 7, the facial nerves abnormally enhance bilaterally (white arrows, $C$ ) and the left ventral pons has an atypical T2 hyperintense focus (black arrow, $D$ ). There is also more ill-defined hyperintensity in the dorsal pons (white arrow, D). 
fore and after intravenous administration of gadobenate dimeglumine (TR, 8.19-8.79 ms; TE, 3.77-4.60 ms; NEX, 1; section thickness, $1 \mathrm{~mm}$ ), axial T2-weighted FSE (TR, 3500-10,000 ms; TE, 80-110 ms; NEX, 1 or 2; section thickness, 2-4 mm), FLAIR imaging (TR, 4800-11,000 ms; TE, 125-330 ms; NEX, 2; section thickness, 1 or $4 \mathrm{~mm}$ ), and axial gradient echo $\mathrm{T} 2^{\star}$-weighted imaging (TR, 550-900 ms; TE, 23-26 ms; flip angle, $18^{\circ}-20^{\circ}$; NEX, 1 or 2; section thickness, 4 or $5 \mathrm{~mm}$ ), or SWI (TR, 18.36-19.34 ms; TE, 25-27 ms; NEX, 1; section thickness, $2 \mathrm{~mm}$ ). Spinal imaging included precontrast axial and sagittal spin-echo T1-weighted (TR, 450-650 ms; TE, 8-10 ms; NEX, 2 or 3; section thickness, 3 mm), T2-weighted (TR, 3000-3500 ms; TE, 100-120 ms; NEX, 2 or 3; section thickness, $3 \mathrm{~mm}$ ), and contrast-enhanced axial and sagittal T1-weighted images. All scans were reviewed independently by 3 fellowship-trained pediatric neuroradiologists.

\section{RESULTS}

\section{Clinical Findings}

Eleven patients met the inclusion criteria. The patient demographics, presenting symptoms, vaccination history, diagnostic work-up, and clinical course have been detailed elsewhere (K.M. et al, unpublished data, 2014) and are summarized in the On-line Table. Briefly, patients presented with neurologic symptoms following a febrile upper respiratory illness, including limb weakness in 8 patients and cranial nerve dysfunction in 9. Involved cranial nerves were VI, VII, IX, X, XI, and XII.

Eight patients tested positive for rhinovirus/enterovirus in the nasopharynx, 4 of which were subtyped as EV-D68 (On-line Table). Findings of enteroviral polymerase chain reaction of the CSF were negative in all patients. Testing for West Nile virus and other arboviruses, herpes viruses, Mycoplasma pneumoniae, parechoviruses, and poliovirus was also negative.

\section{Imaging Findings}

On MR imaging, lesions were identified in the brain stem in 9 patients. The pontine tegmentum was the most common site of brain stem involvement, showing increased T2 signal in 8 cases (Fig 1). A ventral pontine lesion in a ninth case was associated with bilateral facial nerve enhancement (patient 7, Fig 2). Lesions were identified in the midbrain in 2 cases, including the bilateral substantia nigra in 1 case (patient 3, Fig 2), and in the medulla in 5 cases. The dentate nuclei were abnormally T2 hyperintense in 2 cases. No supratentorial lesions were identified.

Ten patients had spinal cord lesions involving the central gray matter (On-line Table and Figs 3 and 4). The spinal cord lesions consisted of either ill-defined, nonenhancing T2 hyperintensity throughout the entire central spinal cord gray matter (Fig 3A-C) or a more well-defined T2 hyperintensity confined to the anterior horn cells (Fig 3D, -E). A single patient, patient 1, underwent both acute (day 3 ) and subacute (day 38) imaging. In this case, the pattern of spinal cord T2 hyperintensity evolved from diffuse central gray matter involvement acutely to an anterior horn cell pattern in the subacute phase (Fig 4). The cases with ill-defined diffuse central gray matter involvement were all imaged earlier than cases with well-defined anterior horn cell signal abnormality (median of 3 days after symptom onset versus 27.5 days, with no overlap between groups). Cord lesions uniformly affected the cer-
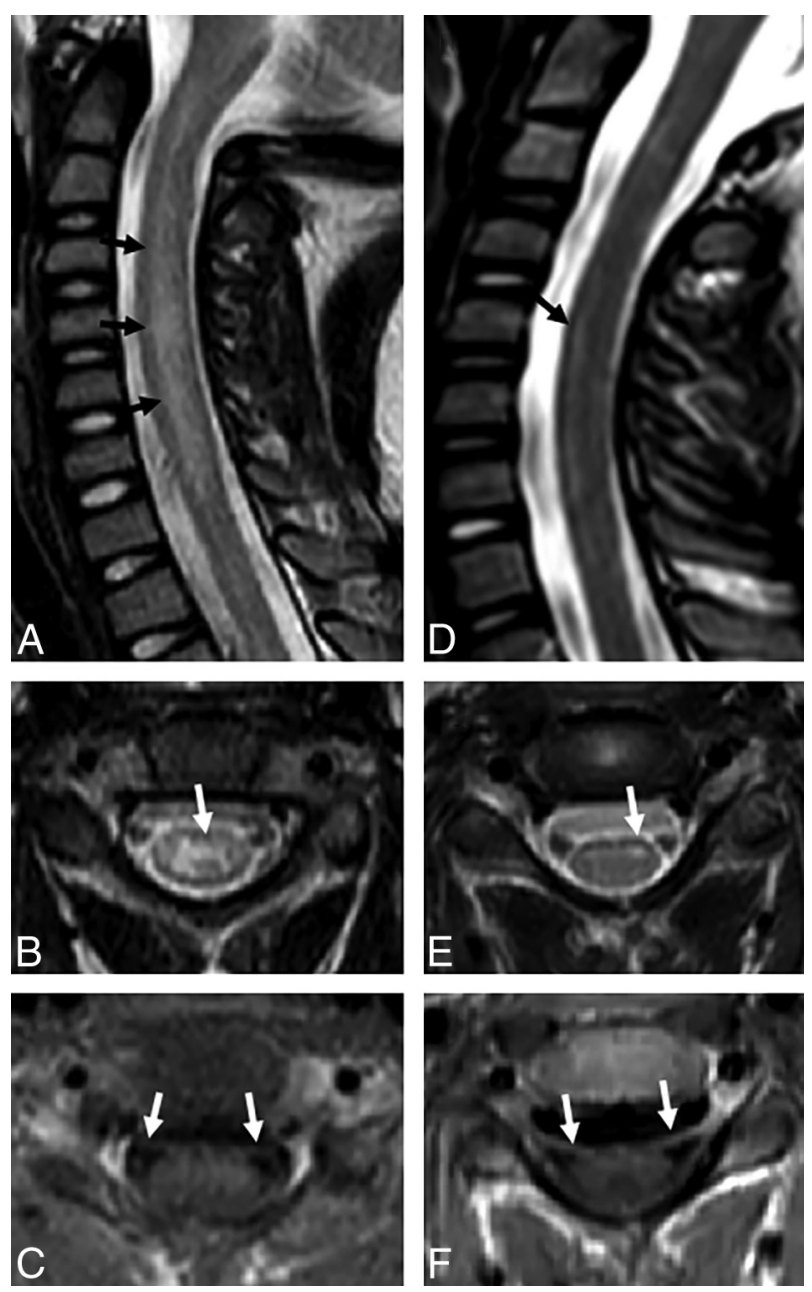

FIG 3. $A-C$, In Patient 2 scanned at 2 days, the central cord hyperintensity is more ill-defined and involves the entire central gray matter (black arrows, $A$; white arrow, $B$ ). Nerve roots do not enhance (white arrows, C). D-F, Patient 8, scanned at 25 days, demonstrates T2 hyperintensity confined to the anterior horn cells asymmetric to the left (black arrow, D; white arrow, E) and nerve root enhancement (white arrows, $F$ ).

vical spine, with decreasing involvement inferiorly. In 4 cases, the lesions extended the entire length of the cord, from the cervicomedullary junction to the conus medullaris (Fig 4). All brain and spinal cord lesions were nonenhancing.

Ventral cauda equina nerve root enhancement occurred in 4 patients (Fig 4), though only 3 had clinical findings of lower extremity weakness. Cervical nerve root enhancement at the levels of spinal cord gray matter T2 hyperintensity and clinical weakness was also detected in 3 patients. Those cases with nerve root enhancement were imaged later than those without (median of 27.5 days after neurologic symptom onset versus 3 days after symptom onset, with no overlap between groups).

\section{DISCUSSION}

Rhombencephalitis and AFP have been reported as uncommon complications of EV-71 infections..$^{9-15}$ Multiple case series have described MR imaging findings in cases of EV-71 neurologic disease, including nonenhancing dorsal brain stem T2 hyperintensity, ${ }^{9,11,15,20-25}$ long-segment T2 hyperintensity of spinal 

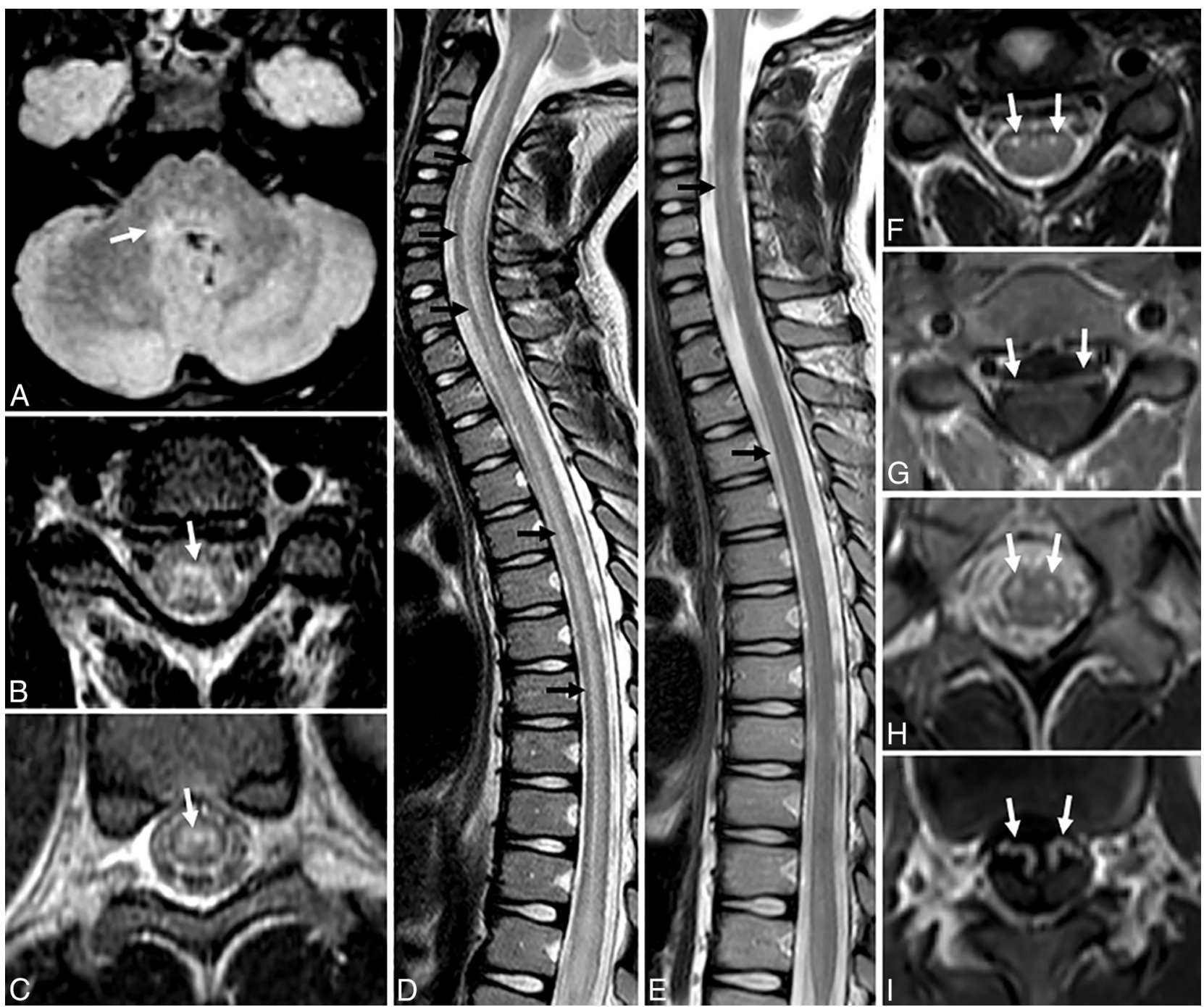

FIG 4. $A-D$, Acute imaging performed at $2-3$ days in patient 1 demonstrates $T 2$ hyperintensity in the right dorsal pons (white arrow, $A$ ) and more ill-defined central gray matter hyperintensity seen more commonly in the acute phase (white arrows, B and C; black arrows, D). E-I, Subacute imaging of the spine performed in the same patient at 38 days demonstrates contraction of the cord T2 hyperintensity to focally involve the anterior horn cells (black arrows, $E$; white arrows, $F$ and $H$ ) and nerve root enhancement of ventral cervical roots (white arrows, $G$ ) and the cauda equina (white arrows, I).

cord anterior horn cells, ${ }^{11,15,20,23,25,26}$ nerve root enhancement, ${ }^{11,15,20-22,25}$ enhancement of anterior horn cells or brain stem, ${ }^{11,15,20,24}$ and substantia nigra involvement. ${ }^{11}$ Similarly, several case reports of MR imaging findings in poliomyelitis have also described dorsal brain stem T2 hyperintensity, ${ }^{16}$ anterior horn cell T2 hyperintensity, ${ }^{17-19,27,28}$ anterior horn cell enhancement, ${ }^{19}$ and substantia nigra T2 hyperintensity. ${ }^{16,17,29}$ Some published cases of AFP caused by West Nile virus have also reported comparable imaging findings of anterior horn-predominant spinal cord lesions and ventral nerve root enhancement. ${ }^{30,31}$ The similarity of the MR imaging findings in this case series to these past reports of virally mediated myelitis suggests a similar direct viral pathogenic effect.

The brain stem was abnormal in most patients in our series, and all of these patients presented with symptoms of cranial nerve dysfunction (Fig 1 and On-line Table). This pattern of clinical involvement in EV-71 neurologic disease has often been described as brain stem encephalitis or rhombencephali- tis. ${ }^{9,11,15,20-25}$ Almost all patients presented with cervical spinal cord T2 hyperintensities, and 9 reported weakness of $\geq 1$ extremity (Fig 3 and On-line Table). This clinical and imaging pattern has been described as AFP in prior case series of EV-71 neurologic disease. ${ }^{11,15,20,23,25,26}$

As mentioned, imaging performed shortly after the onset of CNS symptoms referable to the spinal cord usually demonstrated ill-defined signal abnormality involving the entire central spinal cord gray matter (Fig $3 A-C$ ), while the few patients who were imaged subacutely showed more well-defined signal abnormality confined to the anterior horn cells (Fig $3 D,-E$ ). We speculate that this difference reflects maturation of the disease process, possibly indicating permanent damage, which has been described previously in EV-71 infection. ${ }^{23}$

Other patients with less stereotyped findings include a single patient with diplopia and cranial nerve VI palsies with bilateral substantia nigra T2 hyperintensities (patient 3, Fig 2), which has been described more frequently in poliomyelitis. ${ }^{16,17,29}$ Imaging 
of another patient who presented with bilateral facial nerve palsies revealed a small, nonenhancing ventral pons lesion and bilateral facial nerve enhancement (patient 7, Fig 2). Although facial nerve palsies are frequently reported in EV-71 infections, ${ }^{24}$ to our knowledge, this is the first report of both facial nerve enhancement and a unilateral ventral pontine lesion in the context of suspected enteroviral CNS disease.

Similar to the cases in our cluster, spinal nerve root enhancement has been previously described in cases of EV-71 neurologic disease. ${ }^{11,15,20-22,25}$ Nerve root enhancement is thought to result from either breakdown of the blood-nerve endothelial barrier ${ }^{32}$ or radicular vein enhancement. ${ }^{33}$ In the case of EV-71 neurologic disease and the current series, possible etiologies could include direct viral infection, inflammation related to Wallerian degeneration from anterior horn cell damage, or a postinfectious autoimmune inflammatory process, as is suspected in Guillain-Barrerelated enhancement. ${ }^{34}$

Several imaging features argue against alternative, noninfectious differential diagnoses. None of these patients presented with supratentorial lesions, as are frequently seen in acute disseminated encephalomyelitis. ${ }^{35}$ The restriction of involvement to the central gray matter in our series is not typical of the imaging or clinical findings of idiopathic acute transverse myelitis (admittedly a diagnosis of exclusion), which is typically described as central, extensive spinal cord T2 hyperintensity, swelling, and lesion enhancement. ${ }^{36,37}$ Nerve root enhancement is also not typical of either acute disseminated encephalomyelitis or transverse myelitis. While Guillain-Barre syndrome is characterized by nerve root enhancement, cord and brain stem lesions are not typical features. $^{34}$

Details on treatment of this cohort are described elsewhere (K.M. et al, unpublished data, 2014). To date, most of these patients have residual deficits. Long-term outcomes are unknown. Continued radiographic and neurologic follow-up will be critical to our understanding of the course of this condition.

\section{CONCLUSIONS}

This series of children presenting with neurologic disease after a respiratory illness during an EV-D68 outbreak shares a characteristic pattern of MR imaging findings previously described in past outbreaks of EV-71 neurologic disease, West Nile virus-associated AFP, and poliomyelitis. MR imaging findings in this series include brain stem lesions preferentially involving the pontine tegmentum, spinal cord lesions of the central gray matter acutely and anterior horn cells subacutely, and cranial and spinal nerve root enhancement. The marked similarity of these imaging findings in our cohort of patients to those reported in EV-71, poliomyelitis, and West Nile virus is very suggestive of a direct viral effect. Recognition of these findings is critical to aid clinicians as they consider therapeutic interventions.

Disclosures: Teri Schreiner-UNRELATED: Grants/Grants Pending: Environmental and Genetic Triggers of Pediatric MS, ${ }^{*}$ Comments: This is a National Institutes of Health-funded study. I am in 1 of 14 sites that are participating in the study. Money was paid via contract to the University of Colorado; Payment for Lectures (including service on Speakers Bureaus): Consortium of MS Centers, Comments: I was paid $\$ 500$ (plus travel expenses) to provide a lecture at the annual meeting of the Consortium of MS Centers in May 2014. *Money paid to the institution.

\section{REFERENCES}

1. Committee on the Enteroviruses. Classification of human enteroviruses. Virology 1962;16:501-04

2. Oberste MS, Maher K, Schnurr D, et al. Enterovirus 68 is associated with respiratory illness and shares biological features with both the enteroviruses and the rhinoviruses. J Gen Virol 2004;85(pt 9): 2577-84

3. Centers for Disease Control and Prevention (CDC). Clusters of acute respiratory illness associated with human enterovirus 68: Asia, Europe, and United States, 2008-2010. MMWR Morb Mortal Wkly Rep 2011;60:1301-04

4. Kreuter J, Barnes A. A fatal central nervous system enterovirus 68 infection. Arch Path Lab Med 2011;135:793-96

5. Roux A, Lulu S, Waubrant E, et al. A Polio-like syndrome in California: clinical, radiologic, and serologic evaluation of five children identified by a statewide laboratory over a twelve-month period. Neurology 2014;82(10 suppl):P3.335

6. Midgley C, Jackson MA, Selvarangan R, et al. Severe respiratory illness associated with enterovirus D68: Missouri and Illinois, 2014. MMWR Morb Mortal Wkly Rep 2014;63:798-99

7. CDC Health Alert Network. Acute neurologic illness with focal limb weakness of unknown etiology in children. September 26, 2014. http://www.bt.cdc.gov/han/han00370.asp. Accessed October 6, 2014

8. Pastula DM, Aliabadi N, Haynes AK, et al. Acute neurologic illness of unknown etiology in children: Colorado, August-September 2014. MMWR Morb Mortal Wkly Rep 2014;63;3901-02

9. Wang SM, Liu CC, Tseng HW, et al. Clinical spectrum of enterovirus 71 infection in children in southern Taiwan, with an emphasis on neurological complications. Clin Infect Dis 1999;29:184-90

10. Ooi MH, Wong SC, Lewthwaite $P$, et al. Clinical features, diagnosis, and management of enterovirus 71. Lancet Neurol 2010;9:1097-105

11. Huang CC, Liu CC, Chang YC, et al. Neurologic complications in children with enterovirus 71 infection. $N$ Engl $\mathrm{J}$ Med 1999;341:936-42

12. McMinn P, Stratov I, Nagarajan L, et al. Neurological manifestations of enterovirus 71 infection in children during an outbreak of hand, foot, and mouth disease in Western Australia. Clin Infect Dis 2001;32:236-42

13. McMinn PC. Enterovirus 71 in the Asia-Pacific region: an emerging cause of acute neurological disease in young children. Neurol J Southeast Asia 2003;8:57-63

14. Pérez-Vélez CM, Anderson MS, Robinson CC, et al. Outbreak of neurologic enterovirus type 71 disease: a diagnostic challenge. Clin Infect Dis 2007;45:950-57

15. Chen CY, Chang YC, Huang CC, et al. Acute flaccid paralysis in infants and young children with enterovirus 71 infection: MR imaging findings and clinical correlates. AJNR Am J Neuroradiol 2001;22:200-05

16. Wasserstrom R, Mamourian A, McGary C, et al. Bulbar poliomyelitis: MR findings with pathologic correlation. AJNR Am J Neuroradiol 1992;13:371-73

17. Choudhary A, Sharma S, Sankhyan N, et al. Midbrain and spinal cord magnetic resonance imaging (MRI) changes in poliomyelitis. J Child Neurol 2010;25:497-99

18. Haq A, Wasay M. Magnetic resonance imaging in poliomyelitis. Arch Neurol 2006;63:778

19. Kornreich L, Dagan O, Grunebaum M. MRI in acute poliomyelitis. Neuroradiology 1996;38:371-72

20. Chen F, Li JJ, Liu T, et al. Clinical and neuroimaging features of enterovirus71 related acute flaccid paralysis in patients with handfoot-mouth disease. Asian Pac J Trop Med 2013;6:68-72

21. Lee KY, Lee YJ, Kim TH, et al. Clinico-radiological spectrum in enterovirus 71 infection involving the central nervous system in children. J Clin Neurosci 2014;21:416-20

22. Jang S, Suh S, Ha SM, et al. Enterovirus 71-related encephalomyelitis: usual and unusual magnetic resonance imaging findings. Neuroradiology 2012;54:239-45

23. Shen WC, Chiu $\mathrm{HH}$, Chow $\mathrm{KC}$, et al. MR imaging findings of entero- 
viral encephalomyelitis: an outbreak in Taiwan. AJNR Am J Neuroradiol 1999;20:1889-95

24. Zeng H, Wen F, Gan Y, et al. MRI and associated clinical characteristics of EV71-induced brainstem encephalitis in children with hand-foot-mouth disease. Neuroradiology 2012;54:623-30

25. Li J, Chen F, Liu T, et al. MRI findings of neurological complications in hand-foot-mouth disease by enterovirus 71 infection. Int J Neurosci 2012;122:338-44

26. Shen WC, Tsai C, Chiu H, et al. MRI of Enterovirus 71 myelitis with monoplegia. Neuroradiology 2000;42:124-27

27. Rao DG, Bateman DE. Hyperintensities of the anterior horn cells on MRI due to poliomyelitis. J Neurol Neurosurg Psychiatry 1997;63:720

28. Malzberg M, Rogg J. Poliomyelitis: hyperintensity of the anterior horn cells on MR images of the spinal cord. AJR Am J Roentgenol 1993;161:863-65

29. França MC Jr, Schmutzler KM, Garibaldi SG, et al. Bilateral substantia nigra involvement in vaccine-associated poliomyelitis. Neurology 2006;66:1597-98

30. Kraushaar G, Patel R, Stoneham GW. West Nile virus: a case report with flaccid paralysis and cervical spinal cord MR imaging findings. AJNR Am J Neuroradiol 2005;26:26-29
31. Hainline ML, Kincaid JC, Carpenter DL, et al. West Nile poliomyelitis in a 7-year-old child. Pediatr Neurol 2008;39:350-54

32. Nguyen $C$, Haughton $V$, Ho K, et al. Contrast enhancement in spinal nerve roots: an experimental study. AJNR Am J Neuroradiol 1995; 16:265-68

33. Georgy B, Snow R, Hesselink J. MR imaging of spinal nerve roots: techniques, enhancement patterns, and imaging findings. AJR Am J Roentgenol 1996;166:173-79

34. Yikilmaz A, Doganay S, Gumus $\mathrm{H}$, et al. Magnetic resonance imaging of childhood Guillain-Barre syndrome. Childs Nerv Syst 2010;26: 1103-08

35. Krupp LB, Banwell B, Tenembaum S. Consensus definitions proposed for pediatric multiple sclerosis and related disorders. Neurology 2007;68(16 suppl 2):S7-12

36. Andronikou S, Albuquerque-Jonathan G, Wilmshurst J, et al. MRI findings in acute idiopathic transverse myelopathy in children. $\mathrm{Pe}$ diatr Radiol 2003;33:624-29

37. Wolf VL, Lupo PJ, Lotze TE. Pediatric acute transverse myelitis overview and differential diagnosis. J Child Neurol 2012;27: 1426-36 